\title{
The Rapeutic Effect of Milrinone Combined With Labetalol in Treatment of Severe Cardiopulmonary Failure Caused by Hand, Foot and Mouth Disese
}

\author{
Wenli Li ${ }^{1}$, Huiyuan Cheng ${ }^{2}$, Yuliang Cheng ${ }^{1}$ \\ ${ }^{1}$ Department of Pediatrics, The Maternal and Child Health Hospital of Yichun, Yichun, China \\ ${ }^{2}$ Department of Pediatrics, The People's Hospital of Yichun, Yichun, China
}

Email address:

liwenli1718@163.com (Huiyuan Cheng)

To cite this article:

Wenli Li, Huiyuan Cheng, Yuliang Cheng. The Rapeutic Effect of Milrinone Combined With Labetalol in Treatment of Severe Cardiopulmonary Failure Caused by Hand, Foot and Mouth Disese. American Journal of Pediatrics. Vol. 5, No. 4, 2019 , pp. $183-186$. doi: $10.11648 /$ j.ajp.20190504.12

Received: February 18, 2019; Accepted: July 25, 2019; Published: September 18, 2019

\begin{abstract}
Goal research on therapeutic effect of milrinone combined with Labetalol in treatment of severe cardiopulmonary failure induced by Hand, Foot and Mouth disease. Method: 50 cases of stage 3 pediatric patients with Hand, foot and Mouth disease between January 2010 - February 2014, randomly assigned to treatment group vs control group with 28 cases in each group. No statistical difference $(\mathrm{p}>0.05)$ was found between two groups in terms of patient age, sex and blood pressure, heart rate. Two groups were treated according to guideline on severe Hand, Foot and Mouth diseases, including supplemental oxygen, blood glucose monitoring, vital sign monitoring, anti-virus, methylprednisolone, lowering intracranial pressure, gama- globulin, milrinone (loading dose $50 \mathrm{ug} / \mathrm{kg}$, slow infusion within 10 minutes, maintenance dose $0.25-0.75 \mathrm{ug} / \mathrm{kg} / \mathrm{min}$ using micro-infusion pump). Besides above mentioned regular treatment, treatment group received labetalol $(1-2 \mathrm{mg} / \mathrm{kg} /$ day, oral, q $8 \mathrm{~h}$, Tid, hold when sinus rhythm lower than $20-30 \%$ of baseline, normal bp, heart rate between 120-130). Data on heart rate, bp variation, mechanical ventilation, adverse reaction after 24 hour, 48 hour and 72 hour was recorded by $\mathrm{x} \pm \mathrm{s}$ and compared using t-test. RESULT: Blood pressure is $109 \pm 10.3824$ hours after treatment in treatment group. Heart rate is $132 \pm 15.64$ beats/minutes. Blood pressure is $91 \pm 8.3 \mathrm{mmHg} 48$ hours after treatment. Heart rate is $122 \pm 17.8$ /minutes. Blood pressure is $89 \pm 11.4 \mathrm{mmHg} 72$ hours after treatment. Heart rate is $102 \pm 14.8$ beats/minutes. Blood pressure, heart rate has substantial improvement on treatment group compared with control group. The difference is statistically significant $(\mathrm{p}<0.05)$. Comparing mechanical ventilation rate between two group (mechanical ventilation case before initiation of treatment was not taken into account), treatment group only have 1 case $(0.04 \%)$, whereas control group has 6 cases (24\%). Difference between two group is statistically significant, $\mathrm{X}^{2}=4.37, \mathrm{P}<0.05$. And no adverse reaction was found. Conclusion: Milrinone combined with Labetalol significantly improves cardio-pulmonary function in cardio -pulmonary failure patient caused by severe hand, foot, mouth disease. Combined treatment significantly prevent disease progression and improves prognosis.
\end{abstract}

Keywords: Milrinone, Labetalol, Severe Hand-Foot-Mouth Disease, Cardio-Pulmonary Failure

\section{Introduction}

Hand-foot-mouth disease is a common contagious illness that was caused by enterovirus, transmitted by nasopharyngeal secretions such as saliva, by direct contact, or by fecal-oral transmission [1]. Most cases of HFMD diseases have minor clinical symptoms such as fever, rash on hand/foot/mouth/buttock, whereas small amount of cases can progress to cardio-pulmonary failure, damaging neuro system and even lead to death [2]. The specialist for HFMD in health department have published protocol for clinician (2011 version) to divide EV 71 into 5 stages and recommend milrinone for stage 3 cario-cardio-pulmonary failure [3]. But in recent years, we found that heart rate was poorly controlled in majority cases that is on milrinone treatment. Also it is noted that side effect of milrinone involves fast heart rate in the instructions [4-5]. Since January 2012, Our center achieve significant therapeutic success in treatment of sever HFMD using Milrinone combined with labetalol. Following is the report. 


\section{Materials and Methods}

\subsection{Basic Reference}

From 56 case of severe HFMD that were treated between January $2012 \sim$ February 2014. All the pediatric patients fit the clinical staging criterial made by the health department (Clinical treatment protocol of severe enterovirus EV 71 cases, 1th edition 2014). There are total of 30 male cases, 26 female cases, age ranging from 11 month to 4 years old, average age $2.4 \pm 1.8$ years, course of disease is 1-5 days. There are 4 cases of pulmonary edema patient on admission that requires mechanical ventilation. 56 cases were randomly assigned to treatment group vs control group, 28 cases in each group. There are no statistical significant difference among two groups intern of age, sex and blood pressure, heart rate. See graph 1.

Table 1. Basic information on two groups.

\begin{tabular}{|c|c|c|c|c|c|c|c|}
\hline \multirow{2}{*}{ Group } & \multirow{2}{*}{ Case } & \multirow{2}{*}{$\begin{array}{l}\text { Sex } \\
\text { Male } \\
\end{array}$} & \multicolumn{5}{|c|}{ Age, blood pressure, heart rate mechanical ventilation for pulmonary edema } \\
\hline & & & Female & (Month) & (MmHg) & (times / min) & Case \\
\hline Treatment & 28 & 13 & 15 & $20.0 \pm 3.6$ & $125 \pm 12$ & $169 \pm 24$ & 2 \\
\hline Control & 28 & 17 & 11 & $19.0 \pm 4.5$ & $127 \pm 19$ & $169 \pm 27$ & 3 \\
\hline$\chi^{2}$ OR $t$ & & 0.42 & & 117 & 0.48 & 117 & 0.10 \\
\hline $\mathrm{p}$ & & $<0.05$ & & $<0.05$ & $<0.05$ & $<0.05$ & $<0.05$ \\
\hline
\end{tabular}

\subsection{Method}

Two group were treated by 2011 clinical protocol for HFMD, including vital sign monitoring, blood glucose, methylprednisolone, mannitol, high dose of gamma-globulin, milrinone loading dose of $50 \mathrm{ug} / \mathrm{kg}$ (10 minus slow injection followed by maintenance dose of $0.25 \sim 0.75 \mathrm{ug} / \mathrm{kg}$. $\mathrm{min}$ on micro-infusion pump), ventilation for pulmonary edema. Besides above treatment, patients in treatment group also received milrinone plus labetalol, 1 2 mg/kg. d, Q $8 \mathrm{hr}$, tid. Combined therapy was hold when sinus rhythm decrease $20 \%-25 \%$, reaching to normal range, or stablized at $120-130$ beats/minutes.

\subsection{Observation Criteria}

Observation on blood pressure (SBP) 24, 48, 72 hours after treatment, or improvement on heart rate post treatment, as well as side effect.

\subsection{Statistic Method}

Data was measured by $\bar{x} \pm \mathrm{s}$, comparison between two groups use t- test.

\section{Result}

\subsection{Improvement on Blood Pressure and Heart Rate}

Comparison of Blood Pressure and Heart Rate After Treatment Between Two Groups of Children. The results are shown in table 2. The blood pressure and heart rate of the children in the treatment group and the control group decreased $24 \mathrm{H}, 48 \mathrm{H}$, and $72 \mathrm{H}$ after treatment, but the blood pressure and heart rate decreased more significantly in the treatment group. The difference between the two groups was statistically significant $(\mathrm{P}<0.05)$.

Table 2. Blood pressure, heart rate changes after treatment of $24 \mathrm{H}, 48 \mathrm{H}, 72 \mathrm{H}$.

\begin{tabular}{|c|c|c|c|c|c|c|c|}
\hline \multirow{2}{*}{ Grop } & \multirow{2}{*}{ case } & \multicolumn{2}{|c|}{ Blood pressure(mm Hg) } & \multicolumn{4}{|c|}{ heart rate(times / minutes) } \\
\hline & & $24 \mathrm{H}$ & $48 \mathrm{H}$ & $48 \mathrm{H}$ & $24 \mathrm{H}$ & $48 \mathrm{H}$ & $72 \mathrm{H}$ \\
\hline Treatment & 28 & $103 \pm 11$ & $91 \pm 8$ & $82 \pm 9$ & $132 \pm 16$ & $109 \pm 9$ & $93 \pm 16$ \\
\hline Control & 28 & $109 \pm 10$ & $96 \pm 9$ & $89 \pm 11$ & $141 \pm 16$ & $122 \pm 18$ & $102 \pm 15$ \\
\hline $\mathrm{t}$ & & 281 & 215 & 21 & 211 & 263 & 21 \\
\hline $\mathrm{p}$ & & $<0.05$ & $<0.05$ & $<0.05$ & $<0.05$ & $<0.05$ & $<0.05$ \\
\hline
\end{tabular}

Compare with control group, there are significant improvement on blood pressure, heart rate after $24 / 48 / 72 \mathrm{hr}$ post treatment. The difference is statistically significant $(\mathrm{p}<0.05)$.

\subsection{Cases of the Mechanical Ventilation}

There are 6 cases of mechanical ventilation in control group $(24 \%$ of all cases) vs 1 case in treatment group $(0.04 \%)$. The difference is statistically significant. Cases that start mechanical ventilation since admission was not counted.

\subsection{Side Effect}

There Is No Side Effect Observed in Treatment Group Who Received Labetalol.

\section{Discussion}

\subsection{About Hand, Foot and Mouth Disease}

$88 \%$ of severe and critical hand, foot and mouth disease caused by EV 71 infection [6]. The Ministry of Health's Hand, Foot and Mouth Disease Clinical Expert Group has developed the Expert Consensus for the Clinical Treatment of Severe Cases of Enterovirus EV 71 (1st Edition, 2011). EV 71 infection is divided into 5 phases: Phase 1 - hand, foot and mouth rash; stage 2 - nervous system involvement; stage 3 - cardiopulmonary failure; stage 4 - cardiopulmonary failure; stage 5 - recovery period. The clinical manifestations of the third phase include increased respiratory heart rate, 
cold limbs, cold sweat, increased blood pressure, and wet skin. More opinions are that cardiopulmonary failure is related to autonomic dysfunction or sympathetic hyperactivity caused by brainstem encephalitis. The disease can progress rapidly from stage 3 to stage 4 in a couple of hours if condition is not identified and treated properly. Patient will suffer from rapid breathing, pinky frothy, bloody sputum, pulmonary edema/ hemorrhage as well as low blood pressure, shock and even death.

\subsection{Neurogenic Pulmonary Edema and Failure}

Animal study and clinical research on cranial injury both show strong relationship between severe HFMD and sympathetic hyper function [7-8]. Large amount of catecholamine released precipitate vasoconstriction, increase after load, central redistribution of blood, leading to neurogenic pulmonary edema and failure which is the major cause of the disease. So the key point in treatment stratege to decrease death rate of HFMD should focus on how to prevent the progression toward cardiopulmonary failure [9]. Majority of severe cases of HFMD have strong reactive cardiopulmonary derangement, similar to Clinical symptoms of " autonomic storm", such as arrhythmia, severe hypertension. Chunfeng Liu think that cardiopulmonary failure was caused by brain stem encephalitis leading to the catecholamine surge that is detrimental to heart. After detailed analysis of 9 cases of severe HFMD disease. Sun JF points out that there is significant rise of catecholamine in HFMD patient, further prove the existence of Catecholamine Storm [10]. Studies of autopsy and MRI on HFMD patient done by scholars like binwei Peng, Qiyun Tang, Xiaobi Ling shows that there are widespread inflammation in central nervous system especially in brain stem and spinal cord [11]. Currently it is well recognized that after initial symptom of EV 71 virus invasion such as oral ulcer, vesicular sores, viral pharyngitis, the virus invade central nervous system directly causing damage on brain stem leading to autonomic dysfunction, catecholamine release, further cause vasoconstriction, hypertension. Neurogenic pulmonary edema and hemorrhage.

\subsection{Combination Therapy with Milrinone and Labetalol}

Recommend using vaso-dilation medication Milrinone to treat stage 3 HFMD. Milrinone is synthetic pyridine that can increase cardiac output, decrease pre and after load through vaso-dilation [12]. It is phospho diestrase inhibitor. By inhibting phospho-diesterase, it increases camp concentration in heart muscle cell as well as smooth muscle cell, opens calcium chanel, leading to inotropic, pre/after load reduction and vaso-dialation effect. But it has no inhibitory effect on autonomic hyperfuction and catecholamine release [13]. So labetalol as a selective beta blocker will block beta receptor to prevent Norepinephrine and Epinephrine activation, therefore block ion Chanel abnormality during catecholamine storm, inhibits sodium and calcium influx and potassium efleux. It also work on central nervous system, inhibits autonomic over activation, decrease heart rate, prevent ischemic in heart muscle, reverse adverse effect of catecholamine on heart muscle electrophysiology so to stablize it [14] . It also prevent hypertension and adverse effect of RAAS system. Beta blocker is the only effective treatment on catecholamine storm. So far, there is not enough cases reports on intravenous beta blocker use in pediatric population, we decide to use oral mediation to observe the effect. There is enough evidence to support the use of milrinone and labetalol combined therapy to treat stage 3 HFMD. Our research shows that compared with control group, 28 cases in treatment group that received combined therapy have statistically significant improvement on blood pressure and heart rate 24, 28 and 72 hours after treatment. mechanical ventilation case number difference between two group is also statistically significant. And there is no adverse effect [15].

\section{Conclusion}

In summary, in pre-cardipulmonary failure phase (especially when heart rate and blood pressure is rising), combined therapy that use labetalol and milrinone have significant better therapeutic effect than using milrinone alone. Combined therapy utilized both autonomic inhibition effect of labetalol and vaso-dilation effect of milrinone. And labetalol also inhibit the side effect of reflex sympathetic activation brought by vast-dilation from milrinone. So the overall effect is improve prognosis and decrease death rate.

\section{References}

[1] Hui Yu, early identification of severe case of pedetrician HFMD, China pediatrician magazine 2012, 4: 284-285.

[2] Zhu J, Chen N, Zhou S, Severity of enterovirus A 71 infection in a human SCARB2 knock-in mouse model is dependent on infectious strain and route. Emerging microbes \& infections 2018 Dec; 7 (1); 205.

[3] Li XW Qian SY Wang Q Chinese guidelines for the diagnosis and treatment of hand, foot and mouth disease (2018 edition). World journal of pediatrics; WJP 2018 Oct; 14 (5); 437-447.

[4] Mirand A Peigue-Lafeuille H Clinical characteristics and course of hand, foot, and mouth disease Archives de pediatrie; organe officiel de la Societe francaise de pediatrie 2017 Oct; 24 (10); 1036-1046.

[5] Nanayakkara S Mak V Crannitch K Extended Release Oral Milrinone, CRD-102, for Advanced Heart Failure. The American journal of cardiology 2018 Sep; 122 (6); 1017-1020.

[6] Chang YK, Chen KH, Chen KT Hand, foot and mouth disease and herpangina caused by enterovirus A 71 infections: a review of enterovirus A 71 molecular epidemiology, pathogenesis, and current vaccine development. Revista do Instituto de Medicina Tropical de Sao Paulo 2018 Nov; 60-70.

[7] Nhan LNT Hong NTT Nhu LNT Severe enterovirus A 71 associated hand, foot and mouth disease, Vietnam, 2018: preliminary report of an impending outbreak. Euro surveillance; bulletin Europeen sur les maladies transmissibles $=$ European communicable disease bulletin 2018 Nov; 23 (46). 
[8] Jones E Jones E Jones E Outcomes following severe hand foot and mouth disease: A systematic review and meta-analysis. Forensic science international. Genetics 2018 09; 36176-185.

[9] Pan YZ Song CL Guo YJ Effects of L-carnitine on serum levels of brain natriuretic peptide and $\mathrm{N}$-terminal pro-brain natriuretic peptide and cardiac function in children with severe hand-foot-mouth disease. Zhongguo Dang Dai Er Ke Za Zhi 2018 Aug; 20 (8); 635-640.

[10] Sun JF Li HL Sun BX Correction to: Correlation analysis on serum inflammatory cytokine level and neurogenic pulmonary edema for children with severe hand-foot-mouth disease. European journal of medical research 2018 Jun; 23 (1); 33.

[11] Liang RX Shi XY Zhang YH Etiology and clinical analysis of central nervous system infection caused by Coxsackievirus B 5 in severe hand, foot and mouth disease in Qingdao City, 2013-2014. Zhonghua yu fang yi xue za zhi [Chinese journal of preventive medicine] 2018 Nov; 52 (11); 1168-1172.
[12] Mirand A Peigue-Lafeuille H Clinical characteristics and course of hand, foot, and mouth disease Archives de pediatrie; organe officiel de la Societe francaise de pediatrie 2017 Oct; 24 (10); 1036-1046.

[13] Lomis N Gaudreault F Malhotra M Novel Milrinone Nanoformulation for Use in Cardiovascular Diseases: Preparation and in Vitro Characterization. Molecular pharmaceutics 2018 Jul; 15 (7); 2489-2502.

[14] Patel P Koli D Maitra N Comparison of Efficacy and Safety of Intravenous Labetalol Versus Hydralazine for Management of Severe Hypertension in Pregnancy. Journal of obstetrics and gynaecology of India 2018 Oct; 68 (5); 376-381.

[15] Li XW Qian SY Wang Q Chinese guidelines for the diagnosis and treatment of hand, foot and mouth disease (2018 edition). World journal of pediatrics; WJP 2018 Oct; 14 (5); 437-447. 